Homology, Homotopy and Applications, vol.13(2), 2011, pp.301-313

\title{
STABILITY FOR CLOSED SURFACES IN A BACKGROUND SPACE
}

\author{
RALPH L. COHEN AND IB MADSEN \\ (communicated by Gunnar Carlsson)
}

\begin{abstract}
In this paper we present a new proof of the homological stability of the moduli space of closed surfaces in a simply connected background space $K$, which we denote by $\mathcal{S}_{g}(K)$. The homology stability of surfaces in $K$ with an arbitrary number of boundary components, $\mathcal{S}_{g, n}(K)$, was studied by the authors in a previous paper. The study there relied on stability results for the homology of mapping class groups, $\Gamma_{g, n}$ with certain families of twisted coefficients. It turns out that these mapping class groups only have homological stability when $n$, the number of boundary components, is positive, or in the closed case when the coefficient modules are trivial. Because of this we present a new proof of the rational homological stability for $\mathcal{S}_{g}(K)$, that is homotopy theoretic in nature. We also take the opportunity to prove a new stability theorem for closed surfaces in $K$ that have marked points.
\end{abstract}

\section{Introduction}

In [4], the authors studied stability properties for moduli spaces of surfaces in a simply connected background space $K$. These moduli spaces, denoted $\mathcal{S}_{g, n}(K)$, consist of surfaces $S_{g, n}$ of genus $g$ with $n$ parametrized boundary components, together with a map $f: S_{g, n} \rightarrow K$ which restricts to the boundary $\partial S_{g, n}$ in a prescribed way. As observed in [4] the homotopy type of these moduli spaces do not depend on the choice of this boundary condition, so we will assume $f$ is constant on $\partial S_{g, n}$, mapping it to a fixed basepoint $x_{0} \in K$. The main results of [4] were Theorems 0.1 and 0.3 which together identify the "stable homology" $H_{q}\left(\mathcal{S}_{g, n}(X)\right)$ for $2 q+4 \leqslant g$.

This result was proved using spectral sequence and Postnikov tower arguments, whose main input was a calculation of the stable homology of mapping class groups with certain families of twisted coefficients ("coefficient systems of finite degree"), $H_{*}\left(\Gamma_{g, n} ; V_{g, n}\right)$. Here $\Gamma_{g, n}$ is the mapping class group of orientation-preserving diffeomorphisms of a fixed surface $F_{g, n}$ of genus $g$ and $n$-boundary components that fix the boundary pointwise, $\Gamma_{g, n}=\pi_{0}\left(\operatorname{Diff}\left(F_{g, n}, \partial F_{g, n}\right)\right)$.

The first author was partially supported by NSF grants 0603713 and 0905809 and ERC-AdG 228082. The second author was supported by ERC-AdG 228082 .

Received May 5, 2011, revised July 12, 2011; published on December 19, 2011.

2000 Mathematics Subject Classification: 57R50, 30F99, 57M07.

Article available at http://intlpress .com/HHA/v13/n2/a18 and doi:10.4310/HHA . 2011.v13.n2 a18

Copyright (C) 2011, International Press. Permission to copy for private use granted. 
The goals of the present note are threefold. First, we wish to describe an error in [4] in the group homology calculation for closed surfaces $(n=0)$. Theorem 0.4 of that paper claims that for coefficient systems of appropriately finite degree $d$, $H_{q}\left(\Gamma_{g, n}, V_{g, n}\right)$ is independent of $g$ and $n$, so long as $2 q+d+2 \leqslant g-1$. This theorem is true, and the proof in [4] is correct, so long as $n>0$. However, for closed surfaces, $n=0$, this theorem is not true, unless the the coefficient system has degree 0 (i.e., is constant), which in turn was proved by Harer [6], with this improved stability range proved by Ivanov [7]. A counterexample to this theorem in the closed case is given by a calculation of S. Morita [9], which was pointed out to us by J. Ebert.

The second goal of this paper is to give a new proof of the homological stability theorem for $\mathcal{S}_{g}(K)$ for closed surfaces. This proof is purely homotopy theoretic in nature using homological stability for $\mathcal{S}_{g, n}(K)$ for $n>0$ and some basic, well-known relationships between diffeomorphism groups of closed surfaces, of surfaces with boundary, and those with marked points. We also make heavy use, as we did in [4], of the theorem of Madsen and Weiss [8] proving a generalization of a conjecture of Mumford that can be viewed as establishing these stability results when $X$ is a point.

These new methods allow us to prove the following new theorem, which determines the homotopy type, in a stable range, of the moduli space of closed surfaces in a background space that have marked points. (This is Theorem 1.8 below.) This theorem generalizes the results of Bödigheimer and Tillmann [2].

Theorem 0.1. Let $\mathcal{S}_{g}^{r}(K)$ be the moduli space of closed surfaces in a background space $K$ having genus $g$ and $r$-marked points. There is a map

$$
\alpha^{r}: \mathcal{S}_{g}^{r}(K) \rightarrow \Omega^{\infty}\left(\mathbb{C P}_{-1}^{\infty} \wedge K_{+}\right) \times\left(\mathbb{C P}^{\infty}\right)^{r} \times K
$$

that induces an isomorphism in integral homology, $H_{q}(-; \mathbb{Z})$, for $3 q \leqslant 2 g-2$ and $r>0$.

The third and last goal of the paper is to use the homotopy theoretic techniques mentioned above to give a new proof of Morita's calculation of $H_{1}\left(\Gamma_{g}, H_{1}\left(F_{g}\right)\right)$, which demonstrates the lack of stability for these homology groups.

We remark that since the writing of [4], results there have been generalized in two different ways. In his thesis $[\mathbf{3}]$, S. Boldsen significantly improved the stability range of the homology of mapping class groups, both in the setting of twisted coefficients (when the surface has at least one boundary) and in the setting of closed surfaces when the coefficients are trivial. As we will point out below, Boldsen's improved stability range allows for a similar improvement of the stability range for $H_{*}\left(\mathcal{S}_{g, n}(X)\right)$, $n \geqslant 0$. Secondly, in [10], O. Randal-Williams generalized our result for the homological stability of $\mathcal{S}_{g, n}(K)$ by proving a stability theorem for surfaces having a general tangential structure. In particular his results show that

$$
\alpha: S_{g}(K) \rightarrow \Omega^{\infty}\left(\mathbb{C P}_{-1}^{\infty} \wedge K_{+}\right)
$$

is a homology equivalence in degrees $q$ with $3 q \leqslant g-2$.

We take the opportunity in this note to give a new, short proof of this theorem when one assumes rational coefficients (or coefficients in any field of characteristic zero). This proof is quite easy and shows the relevance of the Becker-Gottlieb transfer map. 
This paper is organized as follows. In Section 1 we review the construction and relationships between the moduli spaces $\mathcal{S}_{g, n}(K)$. We then prove the main new theorem of this paper, Theorem 1.7, which gives homological stability of closed surface spaces with marked points. In Section 2 we describe a relationship with the BeckerGottlieb transfer map and give a short proof of the homological stability of $\mathcal{S}_{g}(K)$. In Section 3 we give a new proof of Morita's calculation [9].

\section{Acknowledgements}

The authors would like to thank J. Ebert for originally pointing out the error in [4]. The first author would also like to thank the Department of Mathematical Sciences at the University of Copenhagen for its hospitality while this work was being carried out.

\section{Spaces of surfaces, and stability of closed surfaces with marked points}

\subsection{Spaces of surfaces and their relationships}

For each $n \geqslant 0$, let $c_{n} \subset \mathbb{R}^{\infty}$ be the image of a fixed embedding $e_{n}: \coprod_{n} S^{1} \hookrightarrow \mathbb{R}^{\infty}$. Let $K$ be a simply connected space with basepoint $x_{0} \in K$. We define moduli spaces $\mathcal{S}_{g, n, m}(K)$ much like in [4]:

$$
\begin{aligned}
\mathcal{S}_{g, n, m}(K)= & \left\{\left(S_{g, n, m}, t, f\right): \text { where } t>0, S_{g, n, m} \subset \mathbb{R}^{\infty} \times[0, t]\right. \text { is a smooth, } \\
& \text { oriented surface of genus } g \text { with } n+m \text { boundary components, so } \\
& \text { that } S_{g, n, m} \cap\left(\mathbb{R}^{\infty} \times\{0\}\right)=c_{n}, \text { and } S_{g, n, m} \cap\left(\mathbb{R}^{\infty} \times\{t\}\right)=c_{m} ; \\
& \left.f: S_{g, n, m} \rightarrow K \text { is a continuous map with } f\left(\partial S_{g, n, m}\right)=x_{0} \in K\right\} .
\end{aligned}
$$

In this description, $S_{g, n, m} \subset \mathbb{R}^{\infty} \times[0, t]$ is embedded "neatly" as in [8]. In particular, the boundary components, which we call $\partial_{\mathrm{in}} S_{g, n, m}=c_{n}$ and $\partial_{\text {out }} S_{g, n, m}=c_{m}$, each have fixed collars. We refer the reader to [8] for details.

Now let $F_{g, n, m}$ be a fixed smooth, oriented surface of genus $g$ with $n+m$ parametrized boundary components; $n$ of these boundary components are designated as "incoming", and the remaining $m$ are designated as "outgoing". Let $\operatorname{Diff}\left(F_{g, n, m}, \partial\right)$ denote the group of orientation-preserving diffeomorphisms that fix the boundary pointwise. As described in $[\mathbf{4}, \mathbf{5}, \mathbf{8}]$, the spaces $\mathcal{S}_{g, n, m}(K)$ are topologized so as to give homeomorphisms

$$
\begin{aligned}
& \mathcal{S}_{g, n, m}(K) \cong \mathbb{R} \times \operatorname{Emb}_{\partial}\left(F_{g, n, m}, \mathbb{R}^{\infty} \times\right. {[0,1]) } \\
& \times \operatorname{Diff(}\left(F_{g, n, m} ; \partial\right) \\
& \operatorname{Map}\left(\left(F_{g, n, m}, \partial\right),\left(K, x_{0}\right)\right) .
\end{aligned}
$$

Here $\operatorname{Emb}_{\partial}\left(F_{g, n, m}, \mathbb{R}^{\infty} \times[0,1]\right)$ denotes the space of neat embeddings that extend $e_{n}: \coprod_{n} S^{1} \rightarrow \mathbb{R}^{\infty} \times\{0\}$ on $\partial_{\text {in }} F_{g, n, m}$, and $e_{m}: \coprod_{m} S^{1} \rightarrow \mathbb{R}^{\infty} \times\{1\}$ on $\partial_{\text {out }} F_{g, n, m}$. Since this embedding space is contractible with a free action of $\operatorname{Diff}\left(F_{g, n, m} ; \partial\right)$, this gives a homotopy equivalence

$$
\mathcal{S}_{g, n, m}(K) \simeq E \operatorname{Diff}\left(F_{g, n, m} ; \partial\right) \times_{\operatorname{Diff}\left(F_{g, n, m} ; \partial\right)} \operatorname{Map}\left(\left(F_{g, n, m}, \partial\right),\left(K, x_{0}\right)\right) .
$$


For $n=m=0$, we ease notation by deleting the subscripts. So

$$
\mathcal{S}_{g}(K) \cong \mathbb{R} \times \operatorname{Emb}\left(F_{g}, \mathbb{R}^{\infty}\right) \times_{\operatorname{Diff}\left(F_{g}\right)} \operatorname{Map}\left(F_{g}, K\right) .
$$

Finally we define the space $\mathcal{S}_{g}^{1}(K)$ to be the space of closed surfaces in the background space $K$ that have a marked point. In other words,

$$
\begin{aligned}
& \mathcal{S}_{g}^{1}(K)=\left\{\left(S_{g}, t, x, f\right): \text { where } t>0, S_{g} \subset \mathbb{R}^{\infty} \times(0, t)\right. \text { is a smooth, oriented } \\
&\left.\quad \text { surface of genus } g, x \in S_{g}, \text { and } f: S_{g} \rightarrow K \text { is a continuous map }\right\} \\
& \cong \mathbb{R} \times \operatorname{Emb}\left(F_{g}, \mathbb{R}^{\infty}\right) \times_{\operatorname{Diff}\left(F_{g}\right)}\left(F_{g} \times \operatorname{Map}\left(F_{g}, K\right)\right) .
\end{aligned}
$$

We now consider the relationships between the spaces, $\mathcal{S}_{g, 0,1}, \mathcal{S}_{g}^{1}$, and $\mathcal{S}_{g}$. First consider the map $p: \mathcal{S}_{g}^{1}(K) \rightarrow \mathcal{S}_{g}(K)$ given by forgetting the marked point. This is the "universal curve". Then by (3) and (4) we have the following:

Proposition 1.1. The map $p: \mathcal{S}_{g}^{1}(K) \rightarrow \mathcal{S}_{g}(K)$ is a fiber bundle with fiber equal to the surface $F_{g}$.

Consider the fiberwise or vertical tangent bundle $T_{v} \mathcal{S}_{g}^{1}(K)$. This is an oriented, two-dimensional vector bundle classified by a map $\tau_{v}: \mathcal{S}_{g}^{1}(K) \rightarrow \mathbb{C P}^{\infty}$. Concretely, this can be defined as follows (following [5] and [8]):

$$
\tau_{v}: \mathcal{S}_{g}^{1}(K) \rightarrow \mathbb{C P}^{\infty}, \quad\left(S_{g}, t, x, f\right) \rightarrow T_{x} S_{g} \subset \mathbb{R}^{\infty} .
$$

Here we are thinking of $\mathbb{C P} \infty$ as the Grassmannian of oriented two-dimensional subspaces of $\mathbb{R}^{\infty}$. Now consider the evaluation map

$$
e: \mathcal{S}_{g}^{1}(K) \rightarrow K, \quad\left(S_{g}, t, x, f\right) \rightarrow f(x) .
$$

Proposition 1.2. There is a homotopy fibration sequence

$$
\mathcal{S}_{g, 0,1}(K) \stackrel{\iota}{\rightarrow} \mathcal{S}_{g}^{1}(K) \stackrel{\tau_{v} \times e}{\longrightarrow} \mathbb{C P}^{\infty} \times K .
$$

Proof. Consider a fixed, neat embedding of the closed disk $e: D^{2} \subset \mathbb{R}^{\infty} \times(-1 / 2,0]$, whose boundary is the fixed embedding of the unit circle $e_{1}: S^{1} \hookrightarrow \mathbb{R}^{\infty} \times\{0\}$. Now let $\left(S_{g, 0,1}, t, f\right) \in \mathcal{S}_{g, 0,1}(K)$. Let $S_{g}=S_{g, 0,1} \cup_{S^{1}} D^{2}$. This is the closed surface one obtains by capping off the boundary $\partial S_{g, 0,1}$. One can also extend the map

$$
f:\left(S_{g, 0,1}, \partial S_{g, 0,1}\right) \rightarrow\left(K, x_{0}\right)
$$

to $S_{g}$ by defining it to be constant (at the basepoint $x_{0}$ ) on $D^{2}$. This construction defines a map

$$
\begin{aligned}
E \operatorname{Diff}\left(F_{g, 0,1}, \partial\right) \times_{\operatorname{Diff}\left(F_{g, 0,1}, \partial\right)} & \operatorname{Map}\left(\left(F_{g, 0,1}, \partial\right),\left(K, x_{0}\right)\right) \\
& \stackrel{q}{\rightarrow} E \operatorname{Diff}\left(F_{g}, y\right) \times_{\operatorname{Diff}\left(F_{g}, y\right)} \operatorname{Map}\left(\left(F_{g}, y\right),\left(K, x_{0}\right)\right),
\end{aligned}
$$

where $y \in F_{g}$ is a marked point and $\operatorname{Diff}\left(F_{g}, y\right)$ is the group of orientation-preserving diffeomorphisms that fix $y \in F_{g}$. We shall need the well-known homotopy fibration 
sequence (see Lemma 1.3 below):

$$
B \operatorname{Diff}\left(F_{g, 0,1}, \partial\right) \rightarrow B \operatorname{Diff}\left(F_{g}, y\right) \stackrel{\tau_{v}}{\longrightarrow} \mathbb{C P}^{\infty},
$$

where, if our model for the classifying space $B \operatorname{Diff}\left(F_{g}, y\right)$ is given by

$$
\operatorname{Emb}\left(F_{g}, \mathbb{R}^{\infty}\right) / \operatorname{Diff}\left(F_{g}, y\right)=\left\{\left(S_{g}, z\right): S_{g} \subset \mathbb{R}^{\infty}, z \in S_{g}\right\},
$$

then $\tau_{v}\left(S_{g}, z\right)=T_{z} S_{g} \subset \mathbb{R}^{\infty}$. Furthermore, since there is an obvious relative homotopy equivalence

$$
\left(F_{g, 0,1}, \partial F_{g, 0,1}\right) \simeq\left(F_{g}, y\right),
$$

we then have an induced equivalence between the mapping spaces

$$
\operatorname{Map}\left(\left(F_{g, 0,1}, \partial\right),\left(K, x_{0}\right)\right) \simeq \operatorname{Map}\left(\left(F_{g}, y\right),\left(K, x_{0}\right)\right),
$$

which is equivariant with respect to the homomorphism $\operatorname{Diff}\left(F_{g, 0,1}, \partial\right) \rightarrow \operatorname{Diff}\left(F_{g}, y\right)$. Therefore there is an induced homotopy fibration sequence

$$
\begin{aligned}
E \operatorname{Diff}\left(F_{g, 0,1}, \partial\right) \times \times_{\operatorname{Diff}\left(F_{g, 0,1}, \partial\right)} & \operatorname{Map}\left(\left(F_{g, 0,1}, \partial\right),\left(K, x_{0}\right)\right) \\
& \stackrel{q}{\rightarrow} E \operatorname{Diff}\left(F_{g}, y\right) \times_{\operatorname{Diff}\left(F_{g}, y\right)} \operatorname{Map}\left(\left(F_{g}, y\right),\left(K, x_{0}\right)\right) \stackrel{\tau_{v}}{\rightarrow} \mathbb{C P}^{\infty} .
\end{aligned}
$$

Now the inclusion of the based maps into the unbased maps

$$
\operatorname{Map}\left(\left(F_{g}, y\right),\left(K, x_{0}\right)\right) \rightarrow \operatorname{Map}\left(F_{g}, K\right)
$$

is equivariant with respect to the action of $\operatorname{Diff}\left(F_{g}, y\right)$. Furthermore, it is the inclusion of the fiber of the equivariant fibration

$$
\operatorname{Map}\left(F_{g}, K\right) \stackrel{e}{\rightarrow} K, \quad f \rightarrow f(y),
$$

where the action of $\operatorname{Diff}\left(F_{g}, y\right)$ on $K$ is trivial. Putting these fibrations together yields a homotopy fibration sequence

$$
\begin{aligned}
E \operatorname{Diff}\left(F_{g, 0,1}, \partial\right) \times_{\operatorname{Diff}\left(F_{g, 0,1}, \partial\right)} & \operatorname{Map}\left(\left(F_{g, 0,1}, \partial\right),\left(K, x_{0}\right)\right. \\
& \stackrel{q}{\rightarrow} E \operatorname{Diff}\left(F_{g}, y\right) \times_{\operatorname{Diff}\left(F_{g}, y\right)} \operatorname{Map}\left(F_{g}, K\right) \stackrel{\tau_{v} \times e}{\longrightarrow} \mathbb{C P}^{\infty} \times K .
\end{aligned}
$$

Now notice that $B \operatorname{Diff}\left(F_{g}, y\right) \simeq E \operatorname{Diff}\left(F_{g}\right) \times_{\operatorname{Diff}\left(F_{g}\right)} F_{g}$. This is seen by observing that by the isotopy extension theorem the natural action of $\operatorname{Diff}\left(F_{g}\right)$ on $F_{g}$ defines a homeomorphism of the homogeneous space $\operatorname{Diff}\left(F_{g}\right) / \operatorname{Diff}\left(F_{g}, y\right)$ with $F_{g}$. More generally, if $X$ is any space with a $\operatorname{Diff}\left(F_{g}\right)$-action, there is an equivalence

$$
E \operatorname{Diff}\left(F_{g}\right) \times_{\operatorname{Diff}\left(F_{g}\right)}\left(F_{g} \times X\right) \simeq E \operatorname{Diff}\left(F_{g}, y\right) \times_{\operatorname{Diff}\left(F_{g}, y\right)} X .
$$

Thus fibration sequence (8) can be rewritten as a homotopy fibration sequence

$$
\begin{aligned}
E \operatorname{Diff}\left(F_{g, 0,1}, \partial\right) \times_{\operatorname{Diff}\left(F_{g, 0,1}, \partial\right)} & \operatorname{Map}\left(\left(F_{g, 0,1}, \partial\right),\left(K, x_{0}\right)\right) \\
& \stackrel{q}{\rightarrow} E \operatorname{Diff}\left(F_{g}\right) \times_{\operatorname{Diff}\left(F_{g}\right)} \operatorname{Map}\left(F_{g}, K\right) \times F_{g} \stackrel{\tau_{v} \times e}{\longrightarrow} \mathbb{C P}^{\infty} \times K .
\end{aligned}
$$

Using (2) and (4), this sequence yields the homotopy fibration sequence

$$
\mathcal{S}_{g, 0,1}(K) \stackrel{\iota}{\rightarrow} \mathcal{S}_{g}^{1}(K) \stackrel{\tau_{v} \times e}{\longrightarrow} \mathbb{C P}^{\infty} \times K .
$$

The homotopy fibration (7) that was used above is a special case of the following well-known lemma: 
Lemma 1.3. Let $M^{d}$ be a closed, oriented smooth manifold of dimension d and let $y \in M$ be a marked point. There is a homotopy fibration sequence

$$
\operatorname{Diff}\left(M, D_{\epsilon}(y)\right) \rightarrow \operatorname{Diff}(M, y) \stackrel{d}{\rightarrow} G L^{+}(d, \mathbb{R}),
$$

where $D_{\epsilon}(y)$ is a small open disk and the map d denotes the differential at $y$.

Proof. We fix a chart $\left(\mathbb{R}^{d}, 0\right) \rightarrow(M, y)$ around $y$, and let $U_{\epsilon}(y)$ be a small open neighborhood of $y$ corresponding to an open $\epsilon$-disk $\mathbb{R}_{\epsilon}^{d} \subset \mathbb{R}^{d}$ around the origin. The covering isotopy theorem shows that we have a Serre fibration

$$
\operatorname{Diff}\left(M, U_{\epsilon}(y)\right) \rightarrow \operatorname{Diff}(M, y) \stackrel{D}{\rightarrow} \operatorname{Emb}\left(\left(\mathbb{R}_{\epsilon}^{d}, 0\right),\left(\mathbb{R}^{d}, 0\right)\right) .
$$

It remains to show that this embedding space is homotopy equivalent to $G L^{+}(d, \mathbb{R})$. This follows because an embedding $f:\left(\mathbb{R}_{\epsilon}^{d}, 0\right) \hookrightarrow\left(\mathbb{R}^{d}, 0\right)$ is homotopic to a linear map via the standard homotopy

$$
f_{t}(u)= \begin{cases}\frac{f(t u)}{t}, & t>0 \\ d f_{0}(u), & t=0 .\end{cases}
$$

Since $\operatorname{Diff}\left(M, D_{\epsilon}(y)\right)$ denotes the group of diffeomorphisms that fixes some open neighborhood $D_{\epsilon}(y)$ pointwise, this completes the proof.

\subsection{Compatibility of the Pontrjagin-Thom maps}

As described in [5], the spaces $\mathcal{S}_{g, n, m}(K)$ form the spaces of morphisms in the topological cobordism category $\mathcal{C}_{2}(K)$ whose objects are nonnegative integers $n \geqslant 0$ and whose morphisms $\operatorname{Mor}_{\mathcal{C}_{2}(K)}(n, m)$ are given by the disjoint union of the spaces

$$
\operatorname{Mor}_{\mathcal{C}_{2}(K)}(n, m)=\coprod_{g \geqslant 0} \mathcal{S}_{g, n, m}(K)
$$

except if $n=m$, in which case $\operatorname{Mor}_{\mathcal{C}_{2}(K)}(n, m)=\left(\coprod_{g \geqslant 0} \mathcal{S}_{g, n, n}(K)\right) \amalg \operatorname{id}_{n}$.

One of the main theorems of [5] was the identification of the homotopy type of the classifying space of the cobordism category $B \mathcal{C}_{2}(K)$ (as well as other, more general, but similarly defined cobordism categories).

Remark. In Section 5 of [5] a category $\mathcal{C}_{d, \theta}$ was defined, given a Serre fibration $\theta: B \rightarrow$ $B O(d)$. If $d=2$ and $\theta$ is the composition of the projection and the orientation cover,

$$
B S O(2) \times K \rightarrow B S O(2) \rightarrow B O(2),
$$

we have $B \mathcal{C}_{2, \theta} \simeq B \mathcal{C}_{2}(K)$. The difference between $\mathcal{C}_{2, \theta}$ and $\mathcal{C}_{2}(K)$ is that the objects of the former are restricted to be a fixed union of circles in $\mathbb{R}^{\infty}$, while in $\mathcal{C}_{2, \theta}$ the objects are arbitrary closed, oriented one-dimensional manifolds. Using the covering isotopy theorem, it follows easily that the corresponding nerves are homotopy equivalent.

The identification of the homotopy type of $B \mathcal{C}_{2}(K)$ used the Pontrjagin-Thom construction to define a functor to the path category of the zero space of a certain Thom spectrum. More specifically, let $\Omega_{0}^{\infty-1}\left(\mathbb{C P}_{-1}^{\infty} \wedge K_{+}\right)$denote the path component of the basepoint in the zero space of the spectrum $\Sigma \mathbb{C P}_{-1}^{\infty} \wedge K_{+}$. Let $\mathcal{P}\left(\Omega_{0}^{\infty-1}\left(\mathbb{C P}_{-1}^{\infty} \wedge\right.\right.$ $\left.K_{+}\right)$) be its path category. Namely, given any connected space $Y$, the path category 
$\mathcal{P}(Y)$ is the topological category whose objects are the points of $Y$ (topologized as the space $Y$ ), and the morphisms between points $y_{1}$ and $y_{2}$ are the space of paths $\mathcal{P}_{y_{1}, y_{2}}(Y)=\left\{(\alpha, r): \alpha:[0, r] \rightarrow Y\right.$ is continuous, satisfying $\alpha(0)=y_{1}, \alpha(r)=$ $\left.y_{2}\right\}$. The following is standard:

Lemma 1.4. Given any connected space $Y$ there is a weak homotopy equivalence $B \mathcal{P}(Y) \simeq Y$.

We record the standard fact that in any connected space $Y$ with basepoint $y_{0}$ there is a homotopy equivalence of the path space with the loop space $\mathcal{P}_{y_{1}, y_{2}}(Y) \simeq \Omega Y$. Such homotopy equivalences are given by choices of fixed paths $\gamma_{1}$ from $y_{0}$ to $y_{1}$ and $\gamma_{2}$ from $y_{2}$ to $y_{0}$. The homotopy equivalence sends a path $\alpha$ from $y_{1}$ to $y_{2}$ to the glued path $\gamma_{1} \circ \alpha \circ \gamma_{2}$ which is a loop at the basepoint $y_{0} \in Y$.

In [5] the Pontrjagin-Thom construction was used to produce a functor

$$
\alpha: \mathcal{C}_{2}(K) \rightarrow \mathcal{P}\left(\Omega_{0}^{\infty-1}\left(\mathbb{C P}_{-1}^{\infty} \wedge K_{+}\right)\right),
$$

and the following theorem was proved:

Theorem $1.5([\mathbf{5}])$. The functor $\alpha: \mathcal{C}_{2}(K) \rightarrow \mathcal{P}\left(\Omega_{0}^{\infty-1}\left(\mathbb{C P}_{-1}^{\infty} \wedge K_{+}\right)\right)$induces a weak homotopy equivalence on the level of classifying spaces

$$
\alpha: B \mathcal{C}_{2}(K) \rightarrow B \mathcal{P}\left(\Omega_{0}^{\infty-1}\left(\mathbb{C P}_{-1}^{\infty} \wedge K_{+}\right)\right) \simeq \Omega_{0}^{\infty-1}\left(\mathbb{C P}_{-1}^{\infty} \wedge K_{+}\right)
$$

Now for each $n \geqslant 0$ let $\gamma_{n} \in \Omega_{0}^{\infty-1}\left(\mathbb{C P}_{-1}^{\infty} \wedge K_{+}\right)$be the image of the functor $\alpha$ on $n \in \mathrm{ObC}_{2}(K)$. The functor $\alpha$ is "pointed" in that $\gamma_{0} \in \Omega_{0}^{\infty-1}\left(\mathbb{C P}_{-1}^{\infty} \wedge K_{+}\right)$is the basepoint. Now consider the map defined on the level of morphisms

$$
\alpha_{n, m}: \coprod_{g \geqslant 0} \mathcal{S}_{g, n, m}(K) \rightarrow \mathcal{P}_{\gamma_{n}, \gamma_{m}}\left(\Omega_{0}^{\infty-1}\left(\mathbb{C P}_{-1}^{\infty} \wedge K_{+}\right)\right) \simeq \Omega^{\infty}\left(\mathbb{C P}_{-1}^{\infty} \wedge K_{+}\right) .
$$

We consider the homotopy compatibility of these maps as $n$ and $m$ vary. We focus our attention on the two cases $\alpha_{0,1}$ and $\alpha_{0,0}$. To compare them we consider a morphism $\left(D^{2}, 1, e\right) \in \mathcal{S}_{0,1,0}(K) \subset \operatorname{Mor}_{\mathcal{C}_{2}(K)}(1,0)$. Here $D^{2}$ is embedded in $\mathbb{R}^{\infty} \times[0,1]$ with its boundary equal to $c_{1} \subset \mathbb{R}^{\infty} \times\{0\}$. The embedding is fixed and has the property that its intersection with $\mathbb{R}^{\infty} \times\left[\frac{1}{2}, 1\right]$ is empty. (In other words its image lies in $\mathbb{R}^{\infty} \times\left[0, \frac{1}{2}\right)$.) The map $e: D^{2} \rightarrow K$ is constant at the basepoint $x_{0} \in K$.

Notice that composing with the morphism $\left(D^{2}, 1, e\right)$ defines a map

$$
\kappa: \mathcal{S}_{g, 0,1}(K) \rightarrow \mathcal{S}_{g}(K) .
$$

This amounts to "capping off the hole" in a surface with one boundary component and extending a map from that surface to the resulting closed surface by letting it be constant on the capping disk.

Now let $\delta=\alpha_{1,0}\left(D^{2}, 1, e\right)$ be the image under the functor $\alpha$ of $\left(D^{2}, 1, e\right)$, viewed as a morphism in $\operatorname{Mor}_{\mathcal{C}_{2}(K)}(1,0)$. The following compatibility theorem is now simply a result of the functoriality of $\alpha$ : 
Theorem 1.6. The following diagram commutes:

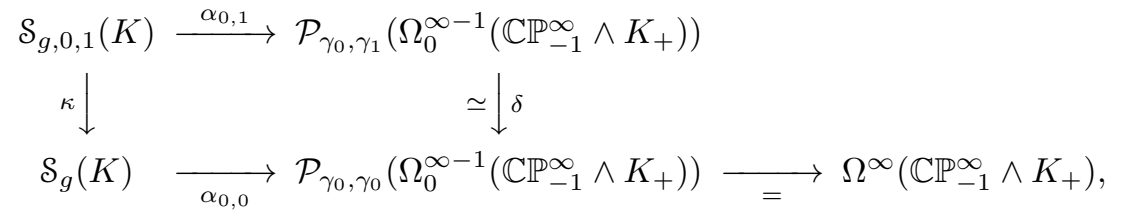

where the right-hand vertical map is concatenation with the fixed path $\delta$.

Comments.

(i) Notice that the map $\kappa$ in this theorem is homotopic to the projection map

$$
\begin{aligned}
E \operatorname{Diff}\left(F_{g, 1}, \partial\right) \times_{\operatorname{Diff}\left(F_{g, 1}, \partial\right)} & \operatorname{Map}\left(\left(F_{g, 1}, \partial F_{g, 1}\right),\left(K, x_{0}\right)\right) \\
& \longrightarrow E \operatorname{Diff}\left(F_{g}\right) \times_{\operatorname{Diff}\left(F_{g}\right)} \operatorname{Map}\left(F_{g}, K\right)
\end{aligned}
$$

defined by capping off the boundary of $F_{g, 1}$ with a disk, and extending a map $f:\left(F_{g, 1}, \partial\left(F_{g, 1}\right)\right) \rightarrow\left(K, x_{0}\right)$ to the closed surface $F_{g}=F_{g, 1} \cup D^{2}$, by defining it on $D^{2}$ to be constant at the basepoint $x_{0} \in K$. It therefore factors, up to homotopy, as the composition,

$$
\mathcal{S}_{g, 0,1}(K) \stackrel{\iota}{\rightarrow} \mathcal{S}_{g}^{1}(K) \stackrel{p}{\rightarrow} \mathcal{S}_{g}(K),
$$

where $\iota$ is as in Proposition 1.1, and $p: \mathcal{S}_{g}^{1}(K) \rightarrow \mathcal{S}_{g}(K)$ forgets the marked point.

(ii) Similar compatibility results between the $\alpha_{n, m}$ 's exist, in general, by capping off various boundary circles. We leave the formulation of these to the reader.

\subsection{Stability with marked points}

We now have the ingredients necessary to prove the main theorem of this section, which gives a stability theorem for the moduli space of closed surfaces in a background space with marked points.

Consider the space $\mathcal{S}_{g}^{1}(K)$. Let $\tilde{\alpha}: \mathcal{S}_{g}^{1}(K) \rightarrow \Omega^{\infty}\left(\mathbb{C P}_{-1}^{\infty} \wedge K_{+}\right)$be the composition

$$
\tilde{\alpha}: \mathcal{S}_{g}^{1}(K) \stackrel{p}{\rightarrow} \mathcal{S}_{g}(K) \stackrel{\alpha}{\rightarrow} \Omega^{\infty}\left(\mathbb{C P}_{-1}^{\infty} \wedge K_{+}\right) .
$$

We then define the map $\alpha^{1}: \mathcal{S}_{g}^{1}(K) \rightarrow \Omega^{\infty}\left(\mathbb{C P}_{-1}^{\infty} \wedge K_{+}\right) \times \mathbb{C P}^{\infty} \times K$ to be the product $\alpha^{1}=\tilde{\alpha} \times \tau_{v} \times e$, with $\tau_{v}$ and $e$ as defined in (5) and (6).

Theorem 1.7. The map

$$
\alpha^{1}: \mathcal{S}_{g}^{1}(K) \rightarrow \Omega^{\infty}\left(\mathbb{C P}_{-1}^{\infty} \wedge K_{+}\right) \times \mathbb{C P}^{\infty} \times K
$$

induces an isomorphism in integral homology, $H_{q}(-; \mathbb{Z})$, for $3 q \leqslant 2 g-2$.

Proof. By Proposition 1.1 and Theorem 1.6, we have the following homotopy commutative diagram of homotopy fibration sequences:

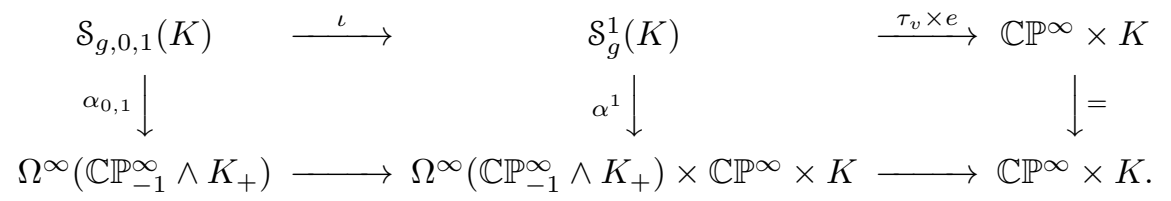

Since the basespace of these fibrations, $\mathbb{C P}^{\infty} \times K$, is simply connected, and since 
$\alpha_{0,1}$ induces a homology isomorphism in this range, then $\alpha^{1}$ induces a homology isomorphism in this range.

We remark that we can also consider moduli spaces of closed surfaces in a background space $K$ with $r$-marked points, $\mathcal{S}_{g}^{r}(K)$. Completely analogous arguments to the above prove the following theorem. We leave the details to the interested reader.

Theorem 1.8. There is a map

$$
\alpha^{r}: \mathcal{S}_{g}^{r}(K) \rightarrow \Omega^{\infty}\left(\mathbb{C P}_{-1}^{\infty} \wedge K_{+}\right) \times\left(\mathbb{C P}^{\infty}\right)^{r} \times K
$$

that induces an isomorphism in integral homology, $H_{q}(-; \mathbb{Z})$, for $3 q \leqslant 2 g-2$.

\section{Relation to the transfer}

Consider the bundle $F_{g} \rightarrow \mathcal{S}_{g}^{1}(K) \stackrel{p}{\rightarrow} \mathcal{S}_{g}(K)$ described in Proposition 1.1. The Becker-Gottlieb transfer map [1] is a map of suspension spectra,

$$
t: \Sigma^{\infty}\left(\mathcal{S}_{g}(K)_{+}\right) \rightarrow \Sigma^{\infty}\left(\mathcal{S}_{g}^{1}(K)_{+}\right)
$$

Its induced map in integral cohomology, $t^{*}: H^{*}\left(\mathcal{S}_{g}^{1}(K)\right) \rightarrow H^{*}\left(\mathcal{S}_{g}(K)\right)$ has the following well-known properties:

Lemma 2.1. For $\beta \in H^{*}\left(\mathcal{S}_{g}(K)\right)$ and $\epsilon \in H^{*}\left(\mathcal{S}_{g}^{1}(K)\right)$,

1. $t^{*}\left(p^{*}(\beta) \epsilon\right)=\beta \cdot t^{*}(\epsilon)$

2. $t^{*}(1)=\chi\left(F_{g}\right) \in H^{0}\left(\mathcal{S}_{g}(K)\right)$.

Here $\chi\left(F_{g}\right)=2-2 g$ is the Euler characteristic.

We will need to relate the transfer map with the Pontrjagin-Thom map $\alpha$ described above. To do this we first need to consider the spectrum map

$$
\tilde{w}: \mathbb{C P}_{-1}^{\infty} \rightarrow \Sigma^{\infty}\left(\mathbb{C P}_{+}^{\infty}\right) .
$$

This can be viewed as "collapsing" the -2-dimensional sphere in $\mathbb{C P}_{-1}^{\infty}$, but more precisely it is induced by a map of Thom spectra,

$$
\tilde{w}: \mathbb{C P}_{-1}^{\infty}=\left(\mathbb{C P}^{\infty}\right)^{-L} \rightarrow\left(\mathbb{C P}^{\infty}\right)^{-L \oplus L}=\Sigma^{\infty}\left(\mathbb{C P}_{+}^{\infty}\right) .
$$

Here $L \rightarrow \mathbb{C P}^{\infty}$ is the canonical oriented two-dimensional bundle, and $-L$ is the corresponding virtual bundle given by its opposite. The exponential notation $X^{\zeta}$ denotes the Thom spectrum of a virtual bundle $\zeta$ over a space $X$. This map of Thom spectra is induced by the inclusion of virtual bundles, $-L \hookrightarrow-L \oplus L$, where, of course, $-L \oplus L$ is the trivial zero-dimensional virtual bundle.

Taking the smash product with the identity produces a similar map of spectra,

$$
\begin{aligned}
\tilde{w}(K): \mathbb{C P}_{-1}^{\infty} \wedge K_{+}=\left(\mathbb{C P}^{\infty} \times K\right)^{-L} \rightarrow\left(\mathbb{C P}^{\infty} \times K\right)^{-L \oplus L} & \\
& =\Sigma^{\infty}\left(\left(\mathbb{C P}^{\infty} \times K\right)_{+}\right) .
\end{aligned}
$$

Here we are thinking of $L \rightarrow \mathbb{C P}^{\infty} \times K$ as the pull-back of the bundle $L \rightarrow \mathbb{C P}^{\infty}$ under the projection map $\mathbb{C P}^{\infty} \times K \rightarrow \mathbb{C P}^{\infty}$. 
Consider the induced map on zero spaces

$$
w(K): \Omega^{\infty}\left(\mathbb{C P}_{-1}^{\infty} \wedge K_{+}\right) \rightarrow \Omega^{\infty} \Sigma^{\infty}\left(\left(\mathbb{C P}^{\infty} \times K\right)_{+}\right),
$$

as well as the cohomology suspension map

$$
\sigma^{*}: H^{*}\left(\mathbb{C P}^{\infty} \times K\right) \rightarrow H^{*}\left(\Omega^{\infty} \Sigma^{\infty}\left(\left(\mathbb{C P}^{\infty} \times K\right)_{+}\right)\right)
$$

Lemma 2.2. Let $\rho=\tau_{v} \times e: \mathcal{S}_{g}^{1}(K) \rightarrow \mathbb{C P}^{\infty} \times K$. Then if $\xi \in H^{*}\left(\mathbb{C P}^{\infty} \times K\right)$,

$$
t^{*} \rho^{*}(\xi)=\alpha^{*} w(K)^{*} \sigma^{*}(\xi) \in H^{*}\left(\mathcal{S}_{g}(K)\right) \text {. }
$$

Here $\alpha=\alpha_{0,0}$ is the map from Theorem 1.6.

Before we prove Lemma 2.2, we show how Lemmas 2.1 and 2.2 together imply the main theorem of this section:

Theorem 2.3.

$$
\alpha^{*}: H^{*}\left(\Omega_{0}^{\infty}\left(\mathbb{C P}_{-1}^{\infty} \wedge K_{+}\right) ; \mathbb{Q}\right) \longrightarrow H^{*}\left(\mathcal{S}_{g}(K) ; \mathbb{Q}\right)
$$

is an isomorphism for $3 * \leqslant 2 g-3$.

Proof. It follows from Lemma 2.1 that $t^{*} \circ p^{*}: H^{*}\left(\mathcal{S}_{g}(K)\right) \rightarrow H^{*}\left(\mathcal{S}_{g}(K)\right)$ is multiplication by $\chi\left(F_{g}\right)=2-2 g$. So with rational coefficients, this composition is an isomorphism so long as $g \neq 1$. (Notice the statement of the theorem is vacuous if $g=1$, so we lose no generality in assuming $g \neq 1$.) It follows that $p^{*}$ is injective, and $t^{*}$ is surjective in rational cohomology.

In the stable range (dimensions less than or equal to $\frac{2}{3} g-1$ ), Theorem 1.7 implies that $\left(\alpha^{1}\right)^{*}: H^{*}\left(\Omega^{\infty}\left(\mathbb{C P}_{-1}^{\infty} \wedge K_{+}\right) \times \mathbb{C P}^{\infty} \times K\right) \rightarrow H^{*}\left(\mathcal{S}_{g}^{1}(K)\right)$ is an isomorphism. Now by the definition of $\alpha^{1}$ given in (9) above, the following diagram commutes:

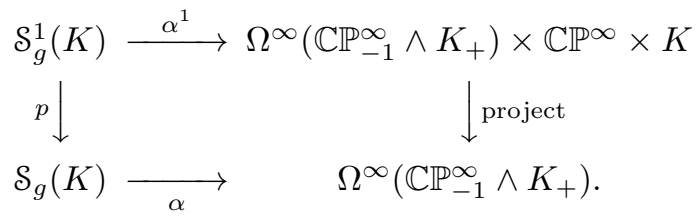

Thus $p^{*} \circ \alpha^{*}$ is injective in this stable range and hence so is $\alpha^{*}$. It remains to show that $\alpha^{*}$ is surjective in this range.

Let $\eta \in H^{*}\left(\Omega_{0}^{\infty}\left(\mathbb{C P}_{-1}^{\infty} \wedge K_{+}\right) ; \mathbb{Q}\right)$ and $\xi \in H^{*}\left(\mathbb{C P}^{\infty} \times K ; \mathbb{Q}\right)$ be classes so that the sum of their dimensions is in the stable range. Then, by definition, $\left(\alpha^{1}\right)^{*}(\eta \otimes \xi)=$ $p^{*} \alpha^{*}(\eta) \cdot \rho^{*}(\xi)$, and by Lemma 2.1 ,

$$
t^{*}\left(\alpha^{1}\right)^{*}(\eta \otimes \xi)=t^{*} p^{*} \alpha^{*}(\eta) \cdot t^{*} \rho^{*}(\xi)=(2-2 g) \alpha^{*}(\eta) \cdot t^{*} \rho^{*}(\xi) .
$$

Lemma 2.2 shows that $t^{*} \rho^{*}(\xi)$ and therefore $t^{*}\left(\alpha^{1}\right)^{*}(\eta \otimes \xi)$ belong to the image of $\alpha^{*}$. Since $t^{*}$ is surjective and $\left(\alpha^{1}\right)^{*}$ is an isomorphism in this range, we conclude that $\alpha^{*}$ is surjective in this range.

It remains to prove Lemma 2.2. Consider again the fiber bundle $F_{g} \rightarrow \mathcal{S}_{g}^{1}(K) \stackrel{p}{\rightarrow}$ $\mathcal{S}_{g}(K)$. The transfer map $t: \Sigma^{\infty}\left(\mathcal{S}_{g}(K)_{+}\right) \rightarrow \Sigma^{\infty}\left(\mathcal{S}_{g}^{1}(K)_{+}\right)$is defined to be the composition

$$
t: \Sigma^{\infty}\left(\mathcal{S}_{g}(K)_{+}\right) \stackrel{\tilde{t}}{\rightarrow} \mathcal{S}_{g}^{1}(K)^{-\tau_{v}} \rightarrow \mathcal{S}_{g}^{1}(K)^{-\tau_{v} \oplus \tau_{v}}=\Sigma^{\infty}\left(\mathcal{S}_{g}^{1}(K)_{+}\right)
$$

where $\tilde{t}: \Sigma^{\infty}\left(\mathcal{S}_{g}(K)_{+}\right) \rightarrow \mathcal{S}_{g}^{1}(K)^{-\tau_{v}}$ is the Pontrjagin-Thom map or "pretransfer". 
The bundle $\tau_{v}$ over $\mathcal{S}_{g}^{1}(K)$ is the vertical tangent bundle classified by $\tau_{v}: \mathcal{S}_{g}^{1}(K) \rightarrow$ $\mathbb{C P}^{\infty}$. The map $\rho=\tau_{v} \times e: \mathcal{S}_{g}^{1}(K) \rightarrow \mathbb{C P}^{\infty} \times K$ induces a map of Thom spectra,

$$
T(\rho): \mathcal{S}_{g}^{1}(K)^{-\tau_{v}} \rightarrow\left(\mathbb{C P}^{\infty} \times K\right)^{-L}=\mathbb{C P}_{-1}^{\infty} \wedge K_{+} .
$$

As in $[\mathbf{5}, \mathbf{8}]$, the map of spectra $\tilde{\alpha}: \Sigma^{\infty}\left(\mathcal{S}_{g}(K)_{+}\right) \rightarrow \mathbb{C P}_{-1}^{\infty} \wedge K_{+}$is defined to be the composition

$$
\tilde{\alpha}: \Sigma^{\infty}\left(\mathcal{S}_{g}(K)_{+}\right) \stackrel{\tilde{t}}{\rightarrow} \mathcal{S}_{g}^{1}(K)^{-\tau_{v}} \stackrel{T \rho}{\longrightarrow}\left(\mathbb{C P}^{\infty} \times K\right)^{-\tau_{v}}=\mathbb{C P}_{-1}^{\infty} \wedge K_{+} .
$$

and $\alpha: \mathcal{S}_{g}(K) \rightarrow \Omega^{\infty}\left(\mathbb{C P}_{-1}^{\infty} \wedge K_{+}\right)$is the adjoint of this map. Using the definition of $\tilde{w}(K)$ given in (10), we then have the following homotopy commutative diagram of spectra:

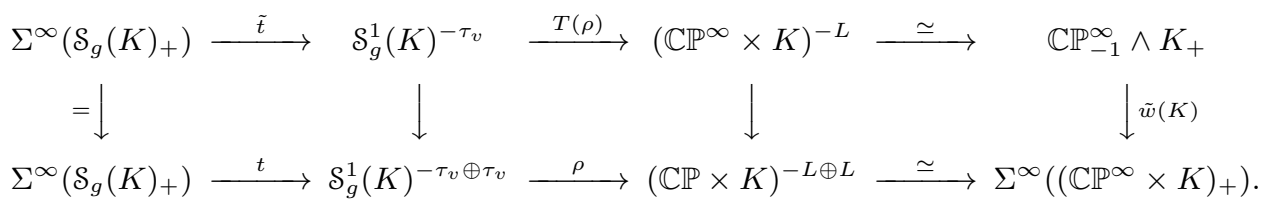

Since the top horizontal composition is $\tilde{\alpha}: \Sigma^{\infty}\left(\mathcal{S}_{g}(K)_{+}\right) \rightarrow \mathbb{C P}_{-1}^{\infty} \wedge K_{+}$, we have that

$$
\tilde{w}(K) \circ \tilde{\alpha} \simeq \rho \circ t: \Sigma^{\infty}\left(\mathcal{S}_{g}(K)_{+}\right) \rightarrow \Sigma^{\infty}\left(\left(\mathbb{C P}^{\infty} \times K\right)_{+}\right) .
$$

So in cohomology, $t^{*} \circ \rho^{*}=\tilde{\alpha}^{*} \circ \tilde{w}(K)^{*}$. Combining this with the general relationship

$$
\tilde{\alpha}^{*} \circ \tilde{w}(K)^{*}=\alpha^{*} \circ w(K)^{*} \circ \sigma^{*}
$$

between maps of suspension spectra and their adjoints, completes the proof of the lemma.

\section{A counterexample to stability for mapping class groups for closed surfaces}

The theorem below, due to S. Morita [9], is a counterexample to Theorem 0.4 of [4] which postulates that $H_{*}\left(\Gamma_{g}, V_{g}\right)$ is independent of the genus $g$ when the $\Gamma_{g}$-module $V_{g}=V\left(F_{g}\right)$ is a coefficient satisfying certain degree conditions. We stress, however, that Theorem 0.4 remains true for surfaces with at least one boundary component: $H_{*}\left(\Gamma_{g, n}, V\left(F_{g, n}\right)\right)$ does have a stable range for $n>0$. Here, as usual, $\Gamma_{g, n}$ is the mapping class group of orientation-preserving diffeomorphisms of an oriented surface $F_{g}$ of genus $g$ with $n$ boundary components. The modules $V\left(F_{g, n}\right)=H_{1}\left(F_{g, n}\right)$ define a coefficient system satisfying the stated degree requirements, but as Morita's theorem below clearly implies, $H_{*}\left(\Gamma_{g}, H_{1}\left(F_{g}\right)\right)$ does not satisfy any stability property. This example was pointed out to the authors by Johannes Ebert.

Theorem 3.1 (Morita [9]).

$$
H_{1}\left(\Gamma_{g} ; H_{1}\left(F_{g}\right)\right) \cong \mathbb{Z} / 2(g-1)
$$

We give a proof of this theorem that is somewhat different in spirit from Morita's proof in [9]. In fact the proof we present below is similar in spirit to the proof of our main Theorem 2.3 above. 
Let $\Gamma_{g}^{1}=\pi_{0}\left(\operatorname{Diff}\left(F_{g}, x_{0}\right)\right)$ and $\Gamma_{g, 1}=\pi_{0}\left(\operatorname{Diff}\left(F_{g}, D^{2}\right)\right.$ be mapping class groups where $D^{2} \subset F_{g}$ is a fixed, small disk with $x_{0} \in D^{2}$. In $\operatorname{Diff}\left(F_{g}, D^{2}\right)$ the diffeomorphisms fix a neighborhood of $D^{2}$ pointwise, and in $\operatorname{Diff}\left(F_{g}, x_{0}\right)$ the diffeomorphisms are only required to fix the point $x_{0}$. Consider the diagram

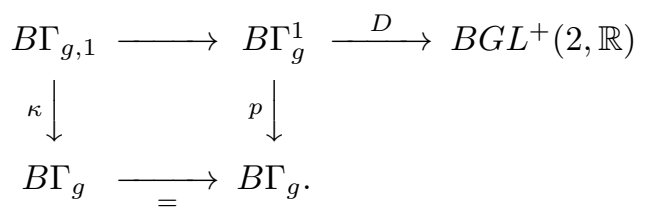

The upper horizontal sequence is the homotopy fibration from Lemma 1.3. The map $\kappa$ caps off the boundary, and $p$ forgets the marked point. The improved Harer stability theorem [6], proved by Boldsen in $[\mathbf{3}]$, asserts that

$$
\kappa_{*}: H_{*}\left(B \Gamma_{g, 1}\right) \rightarrow H_{*}\left(B \Gamma_{g}\right)
$$

is an isomorphism in the range $3 * \leqslant 2 g-2$ and surjective for $3 * \leqslant 2 g$. Since $\kappa$ factors as the composition $\kappa: B \Gamma_{g, 1} \rightarrow B \Gamma_{g}^{1} \stackrel{p}{\rightarrow} B \Gamma_{g}$, in this stable range $p_{*}: H_{*}\left(B \Gamma_{g}^{1}\right) \rightarrow$ $H_{*}\left(B \Gamma_{g}\right)$ has a right inverse $S: H_{*}\left(B \Gamma_{g}\right) \rightarrow H_{*}\left(B \Gamma_{g}^{1}\right)$ satisfying $p_{*} \circ S=$ id. In this range we also have the computation

$$
H_{2}\left(B \Gamma_{g}^{1}\right) \cong H_{2}\left(B \Gamma_{g, 1}\right) \oplus H_{2}\left(B G L^{+}(2, \mathbb{R})\right) \cong \mathbb{Z} \oplus \mathbb{Z} .
$$

The Serre spectral sequence for the fibration $F_{g} \rightarrow B \Gamma_{g}^{1} \stackrel{p}{\rightarrow} B \Gamma_{g}$ has

$$
E_{p, q}^{2}=H_{p}\left(\Gamma_{g} ; H_{q}\left(F_{g}\right)\right) \text {. }
$$

The existence of the section $S: H_{*}\left(B \Gamma_{g}\right) \rightarrow H_{*}\left(B \Gamma_{g}^{1}\right)$ implies that in the stable range there are no nontrivial differential emanating from the baseline, $E_{*, 0}^{2}$. Now we know that

$$
\begin{aligned}
& E_{0,1}^{2}=H_{0}\left(\Gamma_{g} ; H_{1}\left(F_{g}\right)\right)=0 \\
& E_{1,1}^{2}=H_{1}\left(\Gamma_{g} ; H_{1}\left(F_{g}\right)\right) \\
& E_{0,2}^{2}=H_{0}\left(\Gamma_{g} ; H_{2}\left(F_{g}\right)\right)=H_{2}\left(F_{g}\right) \cong \mathbb{Z} .
\end{aligned}
$$

So the only possible differential in total degrees less than or equal to 2 is

$$
d^{2}: E_{2,1}^{2} \rightarrow E_{0,2}^{2} \text {. }
$$

This leads to the exact diagram

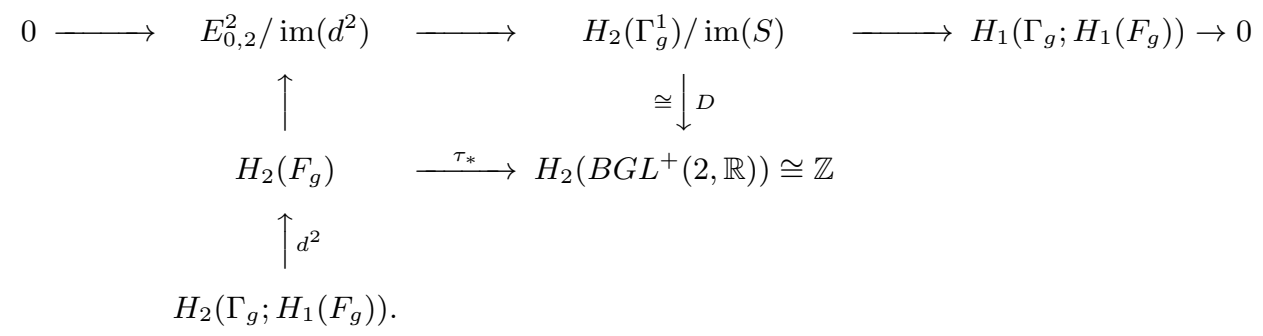

The groups $H_{2}\left(F_{g}\right)$ and $H_{2}\left(B G L^{+}(2, \mathbb{R})\right)$ are each isomorphic to $\mathbb{Z}$, and $\tau: F_{g} \rightarrow$ $\left.B G L^{+}(2, \mathbb{R})\right)$ classifies its tangent bundle and hence induces multiplication by the 
Euler characteristic $2-2 g$ in $H_{2}$. We claim that this implies that the differential $d^{2}$ $=0$. This is because, since $\tau_{*}$ is injective and $D$ is an isomorphism, the commutativity of the above diagram implies $H_{2}\left(F_{g}\right) \rightarrow E_{0,2}^{2} / \mathrm{im}\left(d^{2}\right)$ is injective, which by exactness implies $d^{2}$ is zero. Since, as remarked above, $H_{2}\left(F_{g}\right) \rightarrow E_{0,2}^{2}$ is an isomorphism, the commutativity and exactness of this diagram imply

$$
H_{1}\left(\Gamma_{g} ; H_{1}\left(F_{g}\right)\right) \cong \operatorname{cok}\left(\tau_{*}\right) \cong \mathbb{Z} /(2 g-2) .
$$

\section{References}

[1] J.C. Becker and D.H. Gottlieb, Transfer maps for fibrations and duality, Compositio Math. 33 (1976), no. 2, 107-133.

[2] C.F. Bödigheimer and U. Tillmann, Stripping and splitting decorated mapping class groups (Bellaterra, 1998), Progress in Math. 78 (2001), 47-57, Birkhäuser, Basel.

[3] S. Boldsen, Improved homological stability for the mapping class group with integral or twisted coefficients, Math. Z., to appear.

[4] R.L. Cohen and I. Madsen, Surfaces in a background space and the homology of mapping class groups, Proc. Sympos. Pure Math. 80 (2009), no. 1, 43-76.

[5] S. Galatius, I. Madsen, U. Tillmann and M. Weiss, The homotopy type of the cobordism category, Acta Math. 202 (2009), no. 2, 195-239.

[6] J.L. Harer, Stability of the homology of the mapping class groups of orientable surfaces, Ann. of Math. (2) 121 (1985), no. 2, 215-249.

[7] N.V. Ivanov, On the homology stability for Teichmüller modular groups: closed surfaces and twisted coefficients, Contemporary Math. 150 (1993), 149-194.

[8] I. Madsen and M. Weiss, The stable moduli space of Riemann surfaces: Mumford's conjecture, Ann. of Math. (2) 165 (2007), no. 3, 843-941.

[9] S. Morita, On the homology of the mapping class groups of orientable surfaces with twisted coefficients, Proc. Japan Acad., Ser. A 62 (1986), 148-150.

[10] O. Randal-Williams, Resolutions of moduli spaces and homological stability, preprint, arXiv:0909.4278.

Ralph L. Cohen ralph@math.stanford.edu

Department of Mathematics, Stanford University, Bldg. 380, Stanford, CA 94305, USA

Ib Madsen imadsen@math.ku.dk

Department of Mathematical Sciences, University of Copenhagen, Universitetsparken 5, DK-2100 Copenhagen, Denmark 